\title{
The Total Merger Rate of Compact Object Binaries In The Local Universe
}

\author{
Aleksander Sadowski ${ }^{1}$, Krzysztof Belczynski ${ }^{2,3}$, Tomasz Bulik ${ }^{1,4}$, Natalia Ivanova ${ }^{5}$, Frederic \\ A. Rasio ${ }^{6}$, Richard O'Shaughnessy ${ }^{7}$ \\ as@camk.edu.pl, kbelczyn@nmsu.edu, tb@astrouw.edu.pl, nata@cita.utoronto.ca, \\ rasio@northwestern.edu, oshaughn@gravity.psu.edu
}

\begin{abstract}
Using a population synthesis approach, we compute the total merger rate in the local Universe for double neutron stars, double black holes, and black hole neutron star binaries. These compact binaries are the prime source candidates for gravitational-wave detection by LIGO and VIRGO. We account for mergers originating both from field populations and from dense stellar clusters, where dynamical interactions can significantly enhance the production of double compact objects. For both populations we use the same treatment of stellar evolution. Our results indicate that the merger rates of double neutron stars and black hole - neutron star binaries are strongly dominated by field populations, while merging black hole binaries are formed much more effectively in dense stellar clusters. The overall merger rate of double compact objects depends sensitively on the (largely unknown) initial mass fraction contained in dense clusters $\left(f_{\mathrm{cl}}\right)$. For $f_{\mathrm{cl}} \lesssim 0.0001$, the Advanced LIGO detection rate will be dominated by field populations of double neutron star mergers, with a small but significant number of detections $\sim 20 \mathrm{yr}^{-1}$. However for a higher mass fraction in clusters, $f_{\mathrm{cl}} \gtrsim 0.001$, the detection rate will be dominated by numerous mergers of double black holes
\end{abstract}

\footnotetext{
${ }^{1}$ Nicolaus Copernicus Astronomical Center, Bartycka 18, 00-716 Warszawa, Poland

${ }^{2}$ Los Alamos National Laboratory (Oppenheimer Fellow), Los Alamos, NM, USA

${ }^{3}$ New Mexico State University, Las Cruces, NM, USA

${ }^{4}$ Astronomical Observatory, Warsaw University, Al. Ujazdowskie 4, 00-478, Warsaw, Poland

${ }^{5}$ Canadian Institute for Theoretical Astrophysics, University of Toronto, 60 St. George, Toronto, ON M5S 3H8, Canada

${ }^{6}$ Northwestern University, Dept of Physics and Astronomy, 2145 Sheridan Rd, Evanston, IL 60208

${ }^{7}$ Pennsylvania State University, Department of Physics, 104 Davey Laboratory, University Park, PA 16802
} 
originating from dense clusters, and it will be considerably higher, $\sim 25-300$ $\mathrm{yr}^{-1}$. In addition, we show that, once mergers of double black holes are detected, it is easy to differentiate between systems formed in the field and in dense clusters, since the chirp mass distributions are strikingly different. If significant field populations of double black hole mergers are detected, this will also place very strong constraints on common envelope evolution in massive binaries. Finally, we point out that there may exist a population of merging black hole binaries in intergalactic space.

Subject headings: binaries: close — black hole physics — gravitational waves stars: evolution — globular clusters: general — stellar dynamics

\section{INTRODUCTION}

Gravitational wave astronomy is entering a new era: LIGO (Abramovici et al. 1992) has now taken a full year of data at its design sensitivity; VIRGO (Bradaschia et al. 1990) is nearing completion. Other detectors like GEO or TAMA are also on track. There are well defined plans for improving the LIGO and VIRGO detectors so that, within the next few years, their sensitivity will increase by a factor of up to 30. The list of potential sources for these high-frequency detectors is long and includes supernova explosions, neutron star oscillations, and persistent radiation from rapidly rotating, nonaxisymmetric neutron stars. However, compact object binaries remain the most promising sources. It has been known for some time that their formation may take place in two very different environments: in the galactic field, where their progenitors are massive binaries that evolve in isolation, and in dense star clusters, where they can form at high rates through dynamical interactions.

The properties of the populations of double compact object binaries have been previously investigated using both observational and theoretical approaches. In the typical observational approach, the known compact objects binaries, i.e., radio pulsars in double neutron star (NS-NS) systems, were analyzed in detail. Based on their observed properties and the radio selection effects the properties of the entire population can be reconstructed and the expected merger rate can be calculated (Kim et al. 2005). This approach, however, cannot be extended to black hole - neutron star (BH-NS) binaries and double black hole (BH-BH) binaries since these systems have never been observed. Moreover, we know only one merging NS-NS binary in a globular cluster, and so this method has little power for the population originating in clusters. The second, theoretical, approach is based on numerical

simulations of stellar evolution in field populations, combined with dynamical simulations of star clusters. For field populations some recent examples of theoretical studies include those 
by Belczynski, Kalogera \& Bulik (2002, hereinafter BKB02), Voss \& Tauris (2003), Pfahl et al. (2005), Dewi et al. (2006) and Belczynski et al. (2007a).

The importance of globular cluster evolution for double compact object formation has long been suspected. The fate of BH populations in globular clusters was studied by a number of groups (e.g., Sigurdsson \& Hernquist 1993; Kulkarni, Hut \& McMillan 1993; Portegies Zwart \& McMillan 2000; Merritt et al. 2004). However, previous studies have employed only very simplified treatments of stellar evolution for single stars and binaries in clusters, focusing instead mostly on dynamical interactions. It was pointed out (Phinney et al. 1991; and more recently Grindlay, Portegies Zwart \& McMillan 2006) that the formation of NS-NS and BHNS binaries in clusters is not very efficient and the contribution of clusters to their total merger rate is rather small $(\sim 10-30 \%)$. Although the predicted formation rate of merging double NS has a strong density dependence (Ivanova et al. 2007), even for dense clusters like like 47 Tuc the NS contribution to total cluster merger rates should be overwhelmed by double BH mergers. Such systems are expected to be formed via dynamical interactions in cluster cores (Gultekin, Miller \& Hamilton 2004; O'Leary et al. 2006, 2007). O'Leary et al. (2006, 2007) used realistic initial conditions for their cluster simulations (which included the stellar evolution of massive stars and binaries in the first few Myr of the cluster life) and assumed immediate creation of a BH subcluster. Their calculations did not consider further stellar or binary evolution and neglected the effects of lower-mass stars on BH populations. Their results provide the most up-to-date estimates of $\mathrm{BH}-\mathrm{BH}$ merger rates from globular clusters and the corresponding LIGO detection rates assuming rapid BH segregation into an isolated subcluster. Mackey et al. (2007) investigated the effects of BHs on the structural evolution of globular clusters. They used a realistic initial mass function for single stars and observed rapid mass segregation of the BHs into the cluster core (as expected from the Spitzer instability; see Watters et al. 2000 and references therein). However, this study was based on direct $N$-body simulations that did not include any primordial binaries, even though binaries could affect the mass segregation and subsequent dynamics very significantly.

In this paper we take the next step and calculate the evolution of representative star clusters from the onset of star formation taking into account the large binary fraction for massive stars. We assume that inelastic interactions of hard binaries in the cluster core are effective enough to prevent BHs from completely separating from the rest of the cluster, and we therefore treat the BHs as always well mixed and in thermal equilibrium with other stars in the cluster core. This simplifying assumption is opposite, and complementary, to the one adopted in the models of O'Leary et al. (2006, 2007). Their results and the merger rates calculated in this work give, respectively, lower and upper bounds on the LIGO detection rates. 
In our new models we include both stellar dynamics and full stellar evolution for single and binary stars to predict the merger rate of double compact objects. All stellar populations are evolved and allowed to interact through an entire cluster lifetime ( $\sim 13$ Gyr $)$. Then we combine our estimates for star clusters and the merger rates calculated in O'Leary et al. (2006) with the most recent population synthesis field calculations (Belczynski et al. 2007a) to deduce the total merger rate. In Section 2 we present a description of the stellar binary evolution treatment used and we introduce our model for globular clusters. In Section 3 we present our results and in Section 4 a brief discussion.

\section{MODEL DESCRIPTION}

For field merger rates we use the recent calculations of Belczynski et al. (2007a) obtained with the revised population synthesis code StarTrack (see 2.1). To estimate the merger rates in dense (globular) clusters we employ the dynamical code developed by Ivanova et al. (2005; see also 2.2) to calculate a typical cluster model (see 2.3).

\subsection{Stellar evolution}

Our investigation is based on a population synthesis approach. We use the StarTrack population synthesis code (BKB02), which has been revised and improved significantly over the past few years (Belczynski et al. 2007b).

All stars are evolved based on the metallicity- and wind-mass-loss-dependent models of Hurley, Pols \& Tout (2002), with a few improvements described in BKB02. The main code parameters we use correspond to the standard model presented in Belczynski et al. (2007b). Each star, either single or a binary component, is placed initially on the zeroage main sequence (ZAMS) and then evolved through a sequence of distinct phases: main sequence (MS), Hertzsprung Gap (HG), red giant branch (RG), core He burning (CHeB), asymptotic giant branch (AGB); if a star gets stripped of its H-rich envelope, either through wind mass loss or Roche lobe overflow (RLOF) it becomes a naked helium star (He). The nuclear evolution leads ultimately to the formation of a compact object. Depending on the pre-collapse properties of a star and its composition this may be a white dwarf (WD), a neutron star (NS) or a black hole $(\mathrm{BH})$.

We model the following processes, which can alter the binary orbit and subsequent evolution of the binary components: tidal interactions, magnetic braking, emission of gravitational radiation, and angular momentum changes due to mass loss. Binary components 
may interact through mass transfer and accretion phases. We take into account various modes of mass transfer: wind accretion and Roche lobe overflow (RLOF); conservative and non-conservative; stable or dynamically unstable (leading to common-envelope evolution). The mass transfer rates are calculated from the specific binary configurations and physical properties (masses, evolutionary stages, etc.) of the stars involved. Binary components may loose or gain mass, while the binary orbit may either expand or tighten in response, depending on the particular details of the mass transfer.

Several modifications of the code relevant for double compact object formation were recently introduced: we employ a self-consistent compact object mass calculation (Belczynski et al. 2007b) and spin evolution of BHs in compact binaries is followed (Belczynski et al. 2007c). Additionally, we have taken into account stellar structure of stars crossing Hertzsprung gap and we have pointed out that if such a star initiates common envelope phase it most likely leads to a merger dramatically decreasing formation rates of close double black hole binaries (for more details see Belczynski et al. 2007b).

\subsection{Dynamical interactions}

A full description of our Monte Carlo procedure to treat the dynamics of dense star clusters is given in Ivanova et al. (2005). The most recent updates to the code are described in Ivanova et al. (2006, 2007). The method incorporates a full treatment of binary and single star evolution (as described in 2.1) and allows the dynamical evolution of very large $\left(N \sim 10^{6}\right)$ systems with arbitrary initial binary fractions (e.g., $\sim 50 \%$ ) to be computed in $\sim 1$ month on a modern workstation with 4 processors.

Our modeling of the cluster dynamics is based on several assumptions. We assume that the core number density, $n_{\mathrm{c}}$, and one-dimensional velocity dispersion, $\sigma$, remain constant throughout the evolution (the cluster remains in a constant state of thermal equilibrium for its entire evolution). Typically, the density and "temperature" profiles of a cluster do not change much as long as there are enough binaries remaining to provide support against gravothermal contraction. This state can last for many tens of half-mass relaxation times ( 10 $10^{9} \mathrm{yr}$ for most Galactic globular clusters) as has been shown in many studies (e.g., Fregeau et al. 2003; Giersz \& Spurzem 2003; Hurley \& Shara 2003). It is not clear that the cluster $\mathrm{BH}$ population can in fact remain in thermal equilibrium with other stars in the presence of many binaries. However, as mentioned earlier, we make this simplifying assumption here. The core number density and velocity dispersion are input parameters used to calculate dynamical interaction rates in the cluster core (see below). While all globular clusters have $\sigma_{1 D} \sim 10 \mathrm{kms}^{-1}$, the core density can vary by several orders of magnitude 
reaching $\sim 10^{6} \mathrm{pc}^{-3}$ or even higher in some of the densest clusters (e.g., Harris 1996).

The escape velocity from the cluster core can be estimated from observations as $v_{\mathrm{e}}=$ $2.5 \sigma_{3 D}$ (Webbink 1985), where $\sigma_{3 D}$ is the three-dimensional core velocity dispersion. Following a dynamical interaction or a supernova explosion, any object (single or a binary) that has acquired a recoil speed exceeding $v_{\mathrm{e}}$ is moved outside the cluster where it continues its evolution in isolation.

For computing interactions in the core, the velocities of all objects are assumed to be distributed according to a down-scaled Maxwellian (King 1965), with $f(v)=v^{2} / \sigma(m)^{2}\left(\exp \left(-1.5 v^{2} / \sigma(m)^{2}\right)-\right.$ $\left.\exp \left(-1.5 v_{e}^{2} / \sigma(m)^{2}\right)\right)$ for $v<v_{e}$ and $f(v)=0$ for $v>v_{e}$ with parameters $\sigma(m)=(\langle m\rangle / m)^{1 / 2} \sigma_{3 D}$ (assuming energy equipartition in the core) and $v_{\mathrm{e}}$. In addition, we use $\sigma$ to impose a cut-off for soft binaries entering the core. Any binary with maximum orbital speed $<0.1 \sigma_{3 D}$ is immediately broken into two single stars (Hills 1990).

To model mass segregation in our simulations, we assume that the probability for an object of mass $m$ to enter the core after a time $t_{s}$ follows a Poisson distribution, $p\left(t_{s}\right)=$ $\left(1 / t_{s c}\right) \exp \left(-t_{s} / t_{s c}\right)$, where the characteristic mass-segregation timescale is given by $t_{s c}=$ $10 C(\langle m\rangle / m) t_{\mathrm{rh}}$, Fregeau et al. 2002). Here $t_{\mathrm{rh}}$ is the half-mass relaxation time, which we assume to be constant for a given cluster. We fix $C\langle m\rangle=3 M_{\odot}$, as this value gives, in our model, the best fit for the ratio of core mass to total cluster mass in 47 Tuc.

All objects are allowed to interact dynamically after they have entered the cluster core. We use a Monte-Carlo prescription to decide which pair of objects actually have an interaction during each time step. We consider separately binary-binary and binary-single interactions, as well as single-single encounters (tidal captures and collisions). Tidal captures are treated using the approach described by Portegies Zwart \& Meinen (1993). If the pericenter distance is less than twice the sum of the stellar radii, the encounter is treated as a physical collision and assumed to lead to a merger. Each dynamical interaction involving a binary is calculated using Fewbody, a numerical toolkit for simulating small- $N$ gravitational dynamics that is particularly suited to performing 3-body and 4-body integrations (Fregeau et al. 2004).

\subsection{Standard Cluster Model}

We draw initial masses of single stars and primary stars in binaries using a threecomponent Kroupa (2002) power-law initial mass function with the following exponents: $\alpha_{1}=-0.3$ for $m / M_{\odot}<0.08, \alpha_{2}=-1.3$ for $0.08 \leq m / M_{\odot}<0.5$ and $\alpha_{3}=-2.3$ for 
stars more massive than $0.5 M_{\odot}$. We assume that the minimum object mass is $0.05 M_{\odot} 1$. The stars are allowed to reach masses as high as $150 M_{\odot}$. All stars are formed in a single starburst at the beginning of our simulation. The initial binary mass ratio is assumed to be flat between 0 and 1 . Binary periods are drawn from uniform distribution in $\log _{10} P$ in the range from 0.1 up to $10^{7}$ days. However, binaries with either components filling its Roche lobe are immediately rejected. We assume thermal distribution for eccentricities $(\sim 2 e)$. We set the initial binary fraction to $f_{\mathrm{bi}}=50 \%$ (the number of single stars is equal to the number of binaries). Stars are evolved with low metallicity of $Z=0.001$ appropriate for globular cluster environment (Harris 1996) using standard wind mass loss rates (Hurley et al. 2002) modified by Belczynski (2007b) to include winds from low and intermediate mass main sequence stars. We apply the energy prescription with $\alpha_{C E} \times \lambda=1.0$ for common envelope evolution (Webbink 1984). We assume maximal NS mass to be $2.5 M_{\odot}$. We adopt natal kicks from Hobbs et al. (2005) for NSs, decreased natal kicks for fall-back BHs and no kicks for direct collapse BHs (details can be found in Belczynski et al. 2007b). The merger of two $\mathrm{BH}$ forms a single $\mathrm{BH}$ that receives a recoil kick due to anisotropic emission of gravitational radiation during the merger. We use results of Sopuerta et al. (2007; their best estimate model) to estimate the magnitude of this kick.

Cluster properties are defined by the number of stars, core density, half-mass relaxation time, one-dimensional velocity dispersion and initial core mass fraction. In our model we adopt values typical for a dense globular cluster in our Galaxy. We evolve $10^{6}$ stars (both single and binary stars) with binary fraction of $50 \%$. The total initial mass of the cluster is $4.82 \times 10^{5} M_{\odot}$. This value is higher than the average mass of current Milky Way globular clusters (Gnedin \& Ostriker 1996). However, one has to remember that globular clusters lose a significant fraction of their mass during their evolution due to two-body relaxations, gravitational shocks and stellar evolution. The mass loss can be as high as $50 \%$ in a Hubble time (Fall \& Zhang 2001) and therefore our cluster can be considered typical after a few Gyr of its evolution.

We set the core density to $10^{5} \mathrm{pc}^{-3}$. This choice of density sets the importance of dynamical interactions. The chosen value corresponds to a fairly dense cluster like 47 Tuc. Additionally, we have calculated a cluster model with mass five times lower than the mass of our standard cluster and the merger rate of $\mathrm{BH}-\mathrm{BH}$ binaries per unit mass did not changed significantly (see below). The half-mass relaxation time, which determines the rate of mass segregation, is assumed to be $10^{9} \mathrm{yr}$. The cluster escape velocity is determined by onedimensional velocity dispersion $\sigma_{1 D}$. We set $\sigma_{1 D}=11.54 \mathrm{~km} \mathrm{~s}^{-1}$ which results in $v_{e}=$

\footnotetext{
${ }^{1}$ We allow some objects to reach a mass below the hydrogen-burning limit, $0.08 M_{\odot}$, to avoid skewing the binary mass-ratio distribution.
} 
$50 \mathrm{~km} \mathrm{~s}^{-1}$. We also assume that initially $10 \%$ of the cluster mass is located in the core.

Our calculations with a large fraction of binaries and comprehensive stellar evolution for a very large number of stars in a cluster are computationally expensive. Therefore, we limited this study to 5 independent runs. The obtained merger rates were then averaged. Our results presented here are based on these mean values. To assess the influence of initial cluster mass on the cluster merger rate we performed a calculation for a small cluster (initially

$2 \times 10^{5}$ stars, corresponding to a mass $\sim 10^{5} M_{\odot}$ ) and we found that the merger rates scale down roughly proportionally to mass. We were not able to perform similar calculations for more massive clusters. But one can expect that for massive clusters the rates (again per unit mass) are not lower than for our standard cluster. Therefore, our results can be considered as reasonably robust estimates for the contribution of clusters to the overall merger rates.

\section{RESULTS}

\subsection{Cluster BH-BH formation}

All types of double compact object mergers can be produced in a star cluster. Nevertheless, the most massive objects are most likely participants in dynamical interactions, which leads to the formation of massive tight binaries (Fregeau et al. 2004). Therefore, BH-BH mergers are expected to strongly dominate the overall merger rate from globular clusters (over lighter BH-NS and NS-NS systems).

In Figure 1 we show various evolutionary scenarios leading to $\mathrm{BH}-\mathrm{BH}$ binary mergers. These mergers can be formed either from primordial massive binaries (6\%) or through exchange interactions between single and/or binary stars (94\%). The densities of typical globular cluster cores are high enough to ensure that all BHs interact frequently with other objects. Most of these encounters involve low-mass MS stars (these stars are so numerous that they dominate small cross-section interactions). However, once a close BH-BH binary is formed no star is able, in general, to disrupt it. Dynamical encounters strongly influence the properties of young $\mathrm{BH}$ populations in clusters. The most common interactions, leading to the formation of close BH-BH systems, include encounters of two binaries. Such a binarybinary interaction often leads to a companion exchange (low-mass MS star is replaced by a massive BH). Subsequent interactions (fly-by's) further hardens these systems. At some point the binary becomes tight enough to merge in a short time through angular momentum loss by gravitational radiation. Although all stellar mass BHs are formed very early in the evolution of a cluster (first $\sim 10-20 \mathrm{Myr}$ ), the formation of BH-BH binaries may be extended for a long time due to the ongoing dynamical interactions which create double $\mathrm{BH}$ binaries 
through various evolutionary channels.

It is expected that very few coalescing (in a Hubble time) $\mathrm{BH}-\mathrm{BH}$ binaries can be formed without dynamical interactions in the field (Belczynski et al. 2007a). However, the field population contains a large number of $\mathrm{BH}-\mathrm{BH}$ binaries that are too wide to coalesce in a Hubble time. We find that, even if dynamical interactions are included, globular cluster environments form initially similar populations of BH-BH binaries (mostly wide). However, once the dynamical interactions become more effective in the cluster core, many of these binaries are disrupted (providing single BHs to the cluster population) and a small but significant fraction is hardened by interactions (fly-by's) with other stars (mostly binaries) and evolves to form close BH-BH systems (here about $6 \%$ of the entire cluster population; see Fig. 1).

Let us follow an example of a typical evolution that leads to a $\mathrm{BH}-\mathrm{BH}$ merger. The primordial massive star $\left(M_{\mathrm{ZAMS}} \sim 25 M_{\odot}\right)$ either is formed in or finds its way to the cluster core in a short time. Its evolution leads to a supernova explosion leaving a $\mathrm{BH}$ of mass $M_{\mathrm{BH}, 1} \sim 20 M_{\odot}$. Note that our models are calculated for low metallicity. That leads to reduced mass loss and potential direct $\mathrm{BH}$ formation even at masses as low as 25 Msun. Detailed discussion of $\mathrm{BH}$ mass spectrum at low metallicity was presented by Belczynski et al. (2004). The BH soon becomes a component of a binary as a result of a binary-single exchange. After $40 \mathrm{Myr}$ the $\mathrm{BH}$ exchanges its light binary companion for a MS star of mass $\sim 7 M_{\odot}$. The new companion explodes as a supernova after $87 \mathrm{Myr}$, disrupting the binary. So far the BH and its binaries have undergone $\sim 20$ significant binary-single and 4 binarybinary fly-by interactions in addition to the exchanges. Through the supernova explosion the BH obtains a significant recoil velocity and is ejected from the cluster core into the halo. It takes another $400 \mathrm{Myr}$ for mass segregation to bring it back to the core. At 5 Gyr the $\mathrm{BH}$ acquires again a binary companion. The relatively light companion $\left(M=0.87 M_{\odot} \mathrm{MS}\right.$ star $)$ is soon exchanged for another massive $\mathrm{BH}\left(M_{\mathrm{BH}, 2}=21 M_{\odot}\right.$; this is the last exchange in the history). In the next few Gyr the BH-BH binary is subject to $\sim 80$ binary-single and $\sim 20$ binary-binary fly-by interactions that make the binary orbit harden significantly. After almost 8 Gyr of cluster evolution this BH-BH binary finally merges through gravitationalwave emission. With a mass ratio of merging BHs near unity the merger product (single $\mathrm{BH}$ ) will likely stay in the cluster (i.e., it does not receive a large recoil kick; see $§ 3.2$ ) and is therefore subject to further interactions. 


\subsection{Properties of coalescing $\mathrm{BH}-\mathrm{BH}$ binaries}

Figure 2 presents the number of double $\mathrm{BH}$ mergers as a function of cluster age in our standard cluster model. The solid line shows the numbers of all mergers while the dashed line represents mergers originating from primordial binaries. We show number of mergers per 1 Gyr time bin, so the plot can also be read as a merger rate in $\mathrm{Gyr}^{-1}$. One can see that the overall rate is oscillating around the mean value of $\sim 2.5 \mathrm{Gyr}^{-1}$. The fluctuations are large but in general the merger rate distribution is statistically consistent with the constant rate. Moreover, the globular clusters star formation was assumed to be a $\delta$-function, but if it was spread over some time the merger rate would be even more constant over a long period of time ( Hubble time).

The primordial BH-BH mergers occur in binaries that survived supernova explosions of both components. The BH-BH formation (from primordial stars) happens in the first $\lesssim 20 \mathrm{Myr}$ of the cluster evolution. Thus, one could expect that further tightening due to dynamical encounters and gravitational-wave emission will result in a merger in a relatively short time. In fact, the rate of primordial BH mergers (dashed line in Fig. 22) falls with the age of the cluster, and in particular after $\sim 5$ Gyr of the cluster evolution there is no or very little mergers from primordial binaries.

Few-body interactions favor creation of massive binaries. Therefore, BH-BH binaries that are formed through these dynamical interactions contain most massive BHs. Mergers of such systems are characterized by very high chirp masses. Their distribution is shown in Figure 3. The BH-BH mergers are found with chirp masses of $\sim 10-30 M_{\odot}$ with the peak of a distribution between 18 and $20 M_{\odot}$. These values are significantly higher than expected for the Galactic field BH-BH population $\left(\sim 7 M_{\odot}\right.$; Belczynski et al 2007a). The reason behind a significant change of the chirp mass distribution (from field to cluster population) lies not only in changes of binary mass-ratio distribution caused by dynamical interactions but also in the fact that most massive single black holes are captured into binaries to form massive double black hole binaries. However, it also needs to be noted that black holes in clusters are on average much more massive than in our field simulations as we assume low metallicity of old globular clusters. In particular, for solar metallicity in field populations we form single black holes up to $\sim 10 M_{\odot}$, while for typical globular cluster metallicity single black holes reach $\sim 20 M_{\odot}$ (e.g., Belczynski et al. 2004).

In our models the recoil kick imparted to the product of double BH mergers is calculated as in Sopuerta et al. (2007). These kicks originate from anisotropic gravitational-wave emission during the final merger. Single BHs that are formed in such mergers acquires velocities that are presented in Fig. 4. We find that the velocity distribution is very wide $\sim 10-1000 \mathrm{~km} \mathrm{~s}^{-1}$, and that about $70 \%$ of BHs obtain velocities high enough to leave the 
cluster. It is important to note that the shape of the high end of the recoil velocity distribution is not well determined. Such strong kicks are obtained by high-eccentricity systems which, at present, are not properly modeled (Sopuerta et al. 2007). Nevertheless, there is no doubt that a majority of BHs obtain kicks higher than the cluster ejection velocity $\left(50 \mathrm{~km} \mathrm{~s}^{-1}\right)$ and leave the cluster.

\subsection{LIGO detection rates}

Fig. 5 presents the expected range of detection rates of double $\mathrm{BH}$ mergers for the initial (current stage) and the advanced (expected in 2014) LIGO detector as a function of cluster mass fraction: $f_{\mathrm{cl}}$ - the ratio of stellar mass contained in clusters to the total stellar mass within the reach of the detectors. For both stages of the LIGO detector two lines were presented: the upper limits corresponds to merger rates obtained in this work, the lower limits base on the O'Leary et al. (2006) results.

Our detection rates were obtained assuming Euclidean space geometry with the use of inspiral rates for field $R_{\mathrm{MW}} \approx 15 \mathrm{Myr}^{-1}$ calculated by Belczynski et al. (2007; for a Milky Way-like galaxy: the sum of averaged rates for all double compact mergers in their Model A) and the cluster inspiral rate obtained in this work $\left(R_{\mathrm{cl}, 0}=2.5 \times 10^{-9} \mathrm{yr}^{-1}\right)$ that scaled up to the stellar mass of the Milky Way is: $R_{\mathrm{cl}}=181.5 \mathrm{Myr}^{-1}$. We assumed that the star formation rate in the Galaxy was constant throughout the last $10 \mathrm{Gyr}$ at the rate of $3.5 M_{\odot} \mathrm{yr}^{-1}$, which results in a total stellar mass in the Galactic disk of $M_{\mathrm{MW}}=3.5 \times 10^{10} M_{\odot}$. The detection rates are calculated using the following formula:

$$
\mathcal{R}_{\mathrm{LIGO}}=\rho_{\text {gal }} \frac{4 \pi}{3} d_{0}^{3}\left(\left(1-f_{\mathrm{cl}}\right) \mathcal{M}_{\mathrm{dis}, \mathrm{MW}} \mathcal{R}_{\mathrm{MW}}+f_{\mathrm{cl}} \mathcal{M}_{\mathrm{dis}, \mathrm{cl}} \mathcal{R}_{\mathrm{cl}}\right)
$$

where $f_{\mathrm{cl}}$ is the cluster mass fraction and $\rho_{\text {gal }}=0.01 \mathrm{Mpc}^{-3}$ is the number density of MilkyWay-type galaxies that approximates the mass distribution within the LIGO distance range $d=d_{0}\left(\mathcal{M}_{\mathrm{c}, \mathrm{bhbh}} / \mathcal{M}_{\mathrm{c}, \mathrm{nsns}}\right)^{5 / 6}$, with $d_{0}=18.4,300 \mathrm{Mpc}$ for the initial and advanced LIGO respectively. The distance range estimates were obtained for a binary with two $1.4 M_{\odot}$ neutron stars with chirp mass of $\mathcal{M}_{\mathrm{c}, \mathrm{nnns}}=1.2 M_{\odot}$, and we rescale them for our populations of double BH binaries for given chirp masses $\mathcal{M}_{\mathrm{c}, \text { bhbh }}$. The scaling factor for the cluster population is obtained from

$$
\mathcal{M}_{\text {dis }, \mathrm{cl}}=\left\langle\left(\mathcal{M}_{\mathrm{c}, \mathrm{bhbh}} / \mathcal{M}_{\mathrm{c}, \mathrm{nsns}}\right)^{5 / 2}\right\rangle=1275.0
$$

where the average is taken over all BH-BH mergers we got in the simulation. The adequate factor for the field population is taken from Belczynski et al. $\left(2007 \mathrm{a} ; \mathcal{M}_{\text {dis,Mw }}=71.1\right)$. The specific values for $\rho_{\text {gal }}, d_{0}$ and $\mathcal{M}_{\text {c,nsns }}$ were adopted from O'Shaughnessy et al. (2006). Such 
a procedure is justified instead of a more detailed treatment like the one in Bulik, Belczynski \& Rudak (2004), as there is no significant correlation between the chirp mass and the merger time of binaries.

The merger rates obtained in the O'Leary et al. (2006) work strongly depend on the stage of the cluster evolution. Therefore, to calculate the detection rates, they had to use a cosmological model. It was described in detail in $\S 4.1$ of their work. The authors assumed uniform cluster number density in the universe $\rho_{0}=1 \mathrm{Mpc}^{-3}$, which corresponds to cluster mass fraction $p=0.0014$ (assuming that all clusters resemble our standard model cluster). For the initial stage of LIGO $\left(d_{0}=18.4 \mathrm{Mpc}\right)$ they obtained $\mathcal{R} \sim 0.006 \mathrm{yr}^{-1}$, while for the advanced version of the detector $\left(d_{0}=300 \mathrm{Mpc}\right)$ they got $\mathcal{R} \sim 8 \mathrm{yr}^{-1}$. We combined their results with the merger rates estimations for the field population. The total detection rates as a function of the initial cluster mass fraction, basing on the assumptions and results of the O'Leary et al. (2006) project, are presented in Fig. 5 (the lower limits for both detectors).

The LIGO detection rates presented in Fig. 5 are linearly increasing functions of the cluster mass fraction $f_{\mathrm{cl}}$. Assuming no mass in clusters at all $\left(f_{\mathrm{cl}}=0\right)$ we obtain detection rates as calculated in Belczynski et al. (2007): 0.005yr $r^{-1}$ and $19 y r^{-1}$ for LIGO and advanced LIGO, respectively. Once cluster contribution is included the increase of the detection rates is dramatic. The cluster mass fraction is not well known but is expected at the level $f_{\text {cl }} \gtrsim$ $0.001-0.01$. Therefore, the detection rate of merging compact binaries is found to be $\mathcal{R}_{\text {LIGO }} \gtrsim 0.01-1 \mathrm{yr}^{-1}$ and $\gtrsim 25-3000 \mathrm{yr}^{-1}$ for initial and advanced LIGO, respectively.

Dynamical interactions in the cluster core can result in ejection of a close BH-BH binaries. Most of such events are caused by binary-binary encounters. The ejected systems gain spatial velocity of the order $\sim 100 \mathrm{~km} \mathrm{~s}^{-1}$ and continue evolution in an isolation (i.e., evolution is driven only by emission of gravitational radiation that eventually leads to a merger). Our study shows that $\sim 10 \%$ of BH-BH mergers originating from clusters take place outside cluster boundaries. The coalescence takes $\sim 1 \mathrm{Gyr}$, the time in which a given system can travel $\sim 10-100 \mathrm{kpc}$ and merge in the intergalactic medium. Therefore, we expect that a small but a significant number of advanced LIGO detections of cluster BH-BH mergers will be found far away from host galaxies: $\mathcal{R} \sim 30 y r^{-1}$ and $\sim 300 y r^{-1}$ for $f_{c l}=0.001$ and 0.01 , respectively.

\subsection{Comparison with other studies}

A number of papers estimating the LIGO detection rates have been published recently.

They were based on various methods and assumptions. To estimate the range of total 
merger rates we use the most recent work of O'Leary et al. (2006). They used an approach similar to ours for cluster dynamics (the encounter rate technique). They assumed a uniform cluster number density in the universe $\rho_{0}=1 \mathrm{Mpc}^{-3}$, which corresponds to a cluster mass fraction $p=0.0014$. Their models did not follow stellar evolution so that they had to make several assumptions which differ from this work: (i) BHs were assumed to be completely decoupled from the background cluster stars. (ii) All BH-MS binaries at 11 Myr after the cluster formation were assumed to eventually become BH-BH systems. (iii) All BHs in O'Leary et al. (2006) were introduced initially into the cluster core (they were immediately subject to dynamical interactions). These different assumptions are likely responsible for the qualitatively different results of O'Leary et al. (2006): no BH-MS binaries, rapid BH

subcluster evaporation, and a predicted merger rate rapidly decreasing with time. The detection rates O'Leary et al. (2006) obtained (their Fig. 8) were used as the lower limits for our estimations of the total merger detection rates.

\section{DISCUSSION}

We have presented the ranges of total cosmic merger rate of double compact objects in the local Universe. Our results apply to the distances that will be reached by ground based gravitational-wave detectors such as LIGO or VIRGO ( 300 Mpc for a typical NSNS system). In calculating the rates we have considered formation of close double compact objects in field populations as well as in dense stellar clusters, i.e., globular clusters. The main result of our study shows that the predicted merger rates are too small for detection with the current instruments (i.e., initial LIGO) but are very promising for the upgraded detectors (i.e., advanced LIGO).

We find that field populations dominate the formation of close NS-NS and BH-NS systems. This was already predicted by Phinney (1991) on the basis of observed binary pulsars. Recently Grindlay, Portegies Zwart \& McMillan (2006) have shown that globular clusters can contribute only up to $\sim 20 \%$ to the cosmic NS-NS merger rates. Formation of double compact object systems with NSs is inhibited by the rather large natal kicks NSs receive in supernovae (Hobbs et al. 2005). These lead to (i) disruption of binaries hosting NS progenitors and (ii) ejection of NSs from the cluster. The remaining systems containing a NS interact with heavier stars, and in particular BHs, and through exchange interactions are removed from binaries and do not form double compact objects. Also, the initial cluster mass that we use in our simulations $\left(\sim 5 \times 10^{5} M_{\odot}\right.$, which corresponds to the current average globular cluster mass in our Galaxy; e.g., Meylan \& Heggie 1997, see their Fig. 10.2) is too small to produce a significant number of NS-NS or BH-NS systems. In particular, in our 
cluster simulations, we do not find any mergers of NS-NS nor BH-NS systems. The results for field populations of NS-NS and BH-NS mergers were discussed in detail by Belczynski et al. (2007a). They find (their model A) that field Galactic merger rates are $\sim 15 \mathrm{Myr}^{-1}$ and $\sim 0.1 \mathrm{Myr}^{-1}$ for NS-NS and BH-NS, respectively. In our work we have adopted these rates as the total NS-NS and BH-NS merger rates (field + clusters) since the contribution from clusters is rather small for these systems. As discussed by Belczynski et al. (2007a) these rates are too small for any detection with the initial LIGO, while for advanced LIGO a small but significant number of detections is predicted: $\sim 15 \mathrm{yr}^{-1}$ and $\sim 1 \mathrm{yr}^{-1}$ for NS-NS and $\mathrm{BH}-\mathrm{NS}$, respectively.

Formation of close BH-BH systems that merge in a Hubble time is expected to be very effective in clusters. This was already noted in studies that use realistic initial conditions for the evolution of BH-BH binaries in clusters (e.g., Miller \& Hamilton 2002; Gultekin, Miller \& Hamilton 2004; O'Leary et al. 2006). Here, we have followed the evolution of a realistic average cluster with full stellar evolution and physical treatment of all $\mathrm{BH}-\mathrm{BH}$ binaries, assuming that binary interactions are able to prevent the BHs from separating into an isolated subcluster. We have found that the production of $\mathrm{BH}-\mathrm{BH}$ binary mergers under these assumptions is indeed remarkably effective in dense clusters. The most striking and counter-intuitive difference with other studies is that the predicted $\mathrm{BH}-\mathrm{BH}$ merger rate in clusters is rather constant over long periods of time ( $\sim$ Hubble time). In earlier studies the $\mathrm{BH}$ populations were usually introduced in the cluster core and evolved separately from the other stars in the cluster. That leads to a larger merger rate at early times in the cluster evolution followed by a very rapid drop in the merger rates once BHs are eliminated (through mergers and ejections). However, the assumptions introduced in our work, namely (i) stars that form BHs are initially placed throughout the cluster, and (ii) BHs do not segregate so strongly as to form a completely decoupled subcluster and are instead allowed to interact with other stars in the cluster, make our results qualitatively different. We find that BHs that form in the core, in fact, produce mergers at the early stages of cluster evolution. But later many massive stars that were in the halo and that formed BHs will steadily feed the cluster core with BHs, i.e., continued mass segregation in the cluster halo is providing BHs to the cluster core on long timescales. Additionally, exchange interactions of BHs with unevolved (e.g., main sequence stars) in the core are effective over a long periods of time, and usually lead to formation of close $\mathrm{BH}-\mathrm{BH}$ systems that merge later in the core. A simplified scenario involves two single BHs sinking into the core; each catches a main sequence companion, and then two BH-MS binaries interact, forming a close BH-BH system and two single main sequence stars are released back into the cluster.

Our average cluster merger rate for BH-BH systems is $\sim 3 \mathrm{Gyr}^{-1}$ (see Figure 2) for our simulated cluster with mass $M_{\mathrm{cl}}=4.8 \times 10^{5} M_{\odot}$. If we scaled this rate to the mass of the 
galactic disk $\left(M_{\mathrm{MW}}=3.5 \times 10^{10} M_{\odot}\right)$, then the $\mathrm{BH}-\mathrm{BH}$ merger rate would come up to $\sim 180$ $\mathrm{Myr}^{-1}$ (i.e., this is the expected $\mathrm{BH}-\mathrm{BH}$ merger rate if the entire mass of our Galactic disk were contained in dense globular clusters). We should compare this rate to the Galactic field BH-BH merger rate, $0.025 \mathrm{Myr}^{-1}$ (Belczynski et al. 2007a). It is clear that the production of $\mathrm{BH}-\mathrm{BH}$ mergers is much more efficient (by $\sim 3-4$ orders of magnitude) in clusters as compared to field evolution 2 .

To combine our predicted cluster rates with the field rates we need to know the initial stellar mass fraction contained in clusters. If we look at the Galactic globular clusters we find that they contain about 0.001 of the total mass in stars found in the field $\left(f_{\mathrm{cl}}=0.001\right)$. However, it is reasonable to expect that many clusters may have been completely destroyed and that the clusters we see today were initially more massive and lost significant mass through evaporation (e.g., Vesperini 1998; Joshi et al. 2001). Although we present our predictions for the entire range of plausible $f_{\mathrm{cl}}$ values, we consider that the most reasonable estimate is still $f_{\mathrm{cl}} \gtrsim 0.001$. While we do not have reliable mass estimates for globular clusters in elliptical galaxies, it has been shown that the specific frequency of GCs per galaxy luminosity in ellipticals is significantly (about an order of magnitude) higher than in spiral galaxies (Kim \& Fabbiano 2004). Moreover, elliptical galaxies are on average more massive than spiral galaxies. Therefore, an upper limit on the initial mass fraction contained in all clusters, although highly uncertain, can probably be set to $f_{\mathrm{cl}} \lesssim 0.01$.

The total cosmic merger rate for $\mathrm{BH}-\mathrm{BH}$ systems is then strongly dependent on the mass contained in globular clusters. For a small fraction $\left(f_{\mathrm{cl}}=0.001\right)$ we find the merger rate per Milky Way in our model to be $\sim 0.2 \mathrm{Myr}^{-1}$, while for a larger fraction $\left(f_{\mathrm{cl}}=0.01\right)$ the merger rate is $\sim 2 \mathrm{Myr}^{-1}$. At this point we can also estimate the detection rate for a given GW detector. We must keep in mind that the average chirp mass of field $\mathrm{BH}-\mathrm{BH}$ binaries is much smaller $\left(M_{\mathrm{c}} \sim 7 M_{\odot}\right)$ than for cluster $\mathrm{BH}-\mathrm{BH}$ mergers $\left(M_{\mathrm{c}} \sim 20 M_{\odot}\right)$. This allows us to observe cluster $\mathrm{BH}-\mathrm{BH}$ mergers in a much larger volume and their relative contribution to the detection rate is greater than indicated simply by the merger rates (see $\S$ 3.3). One could also imagine forming of few very massive BHs (up to $\sim 100 M_{\odot}$ ) in the cluster (e.g., through mergers of massive binaries; Belczynski et al. 2006) which could form BH-BH mergers characterized by extremely high chirp masses. Such mergers would be detectable from much greater distances, making the observed rates even higher.

The predicted ranges of total detection rates, for all types of double compact objects, are presented in Figure 5 as a function of cluster contribution. The most likely range of values

\footnotetext{
${ }^{2}$ We have used the rate from the same evolutionary model used for field population (model A of Belczynski et al. 2007a) as it was employed in our standard cluster model.
} 
for this parameter $\left(f_{\mathrm{cl}} \sim 0.001 \div 0.01\right)$ is marked with the vertical shaded area in Figure 5 , For very low cluster contributions $\left(f_{\mathrm{cl}}=0.0001\right)$ the detection rates correspond to mergers coming only from field populations and are adopted from the reference model of Belczynski et al. (2007b; their model A). With increasing cluster contribution we see a drastic increase in predicted detection rates. This increase is connected to the very effective production of BH-BH mergers in clusters as discussed above. For advanced LIGO the detection rates could be as high as $\sim 25-3000 \mathrm{yr}^{-1}$ and are higher by more than an order of magnitude than the rates just for field populations. The total rates are dominated by dynamically formed $\mathrm{BH}-\mathrm{BH}$ mergers in dense stellar clusters. If advanced LIGO does not observe this population of $\mathrm{BH}-\mathrm{BH}$ mergers it will put strong constraints on the initial stellar mass fraction contained in dense stellar clusters.

The production of $\mathrm{BH}-\mathrm{BH}$ mergers in the field is inhibited by the process identified in Belczynski et al. (2007b): many potential BH-BH progenitors evolve through a common envelope phase while the donor is evolving through the Hertzsprung gap. Such a common envelope leads most likely to a merger and aborts potential formation of a BH-BH system (because, in the Hertzsprung gap, the star has not yet developed a clear core-envelope structure and the inspiral does not stop before complete merger of the two interacting stars). If this current understanding of the common envelope phase is correct, we do not expect detection of more than a few field BH-BH mergers per year. However, if the progenitors somehow survive this phase, we could then expect up to $\sim 100$ detections of field BH-BH mergers per year by Advanced LIGO. Since the chirp mass distribution of the field and cluster populations are so different (see Figure 3 and Figure 4 of Belczynski et al. 2007b) it would be easy to tell the two populations apart. This would, in turn, allow us to both (i) derive the initial mass fraction in clusters, and (ii) constrain the fate of massive binary systems going through a common envelope phase. This result highlights the importance of the chirp mass distribution as a diagnostic tool in gravitational wave astronomy (Bulik \& Belczynski, 2003; Bulik, Belczynski, \& Rudak, 2004).

AS, KB and TB acknowledge support from KBN grants 1P03D02228 and 1P03D00530. FAR acknowledges support from NSF Grant PHY-0601995 and NASA Grant NNG06GI62G to Northwestern University. We also thank anonymous referee for practical report that helped us in presentation of our results.

\section{REFERENCES}

Abramovici, A., et al. 1992, Science, 256, 325 
Belczynski, K., Sadowski, A., Rasio, F. A. 2004, ApJ, 611, 1068

Belczynski, K., Kalogera, V., Rasio, F. A., Taam, R. E., Zezas, A., Bulik, T., Maccarone, T. J., Ivanova, N. 2007b, ApJS, in press (astro-ph/0511811)

Belczynski, K., Sadowski, A., Rasio, F. A., Bulik, T. 2006, ApJ, 650, 303

Belczynski, K., Taam, R. E., Kalogera, V., Rasio, F. A., Bulik, T. 2007a, ApJ, accepted (astro-ph/0612032)

Belczynski, K., Taam, R. E., Rantsiou, E., van der Sluys, M. 2007c, ApJ, submitted (astro-ph/0703131)

Bradaschia, C., et al. 1990, Nuclear Instruments and Methods in Physics Research A, 289, 518

Bulik, T., Belczynski, K., 2003, ApJ, 589, L37

Bulik, T., Belczynski, K., Rudak, B., 2004, A\&A, 415, 407

Dewi, J. D. M., Podsiadlowski, Ph., Sena, A. 2006, MNRAS, 368, 1742

Fall, S. M. \& Zhang, Q. 2001, ApJ, 561, 751

Fregeau, J. M., Cheung, P., Portegies Zwart, S. F. \& Rasio, F. A. 2004, MNRAS, 352, 1

Fregeau, J. M., Gurkan, M. A., Joshi, K. K. \& Rasio, F. A. 2003, ApJ, 593, 772

Fregeau, J. M., Joshi, K. J., Portegies Zwart, S. F. \& Rasio, F. A. 2002, ApJ, 570, 171

Giersz, M. \& Spurzem, R. 2003, MNRAS, 343, 781

Gnedin, O. Y. \& Ostriker, J. P. 1996, ApJ, 474, 223

Grindlay, J., Portegies Zwart, S., McMillan, S. 2006, Nature, 2, 116

Gultekin, K., Miller, M., \& Hamilton, D. 2004, ApJ, 616, 221

Harris, W. E. 1996, AJ, 112, 1487

Hobbs, G.; Lorimer, D. R.; Lyne, A. G.; Kramer, M. 2005, MNRAS, 360, 974

Hills, J. G. 1990, AJ, 99, 979

Hurley, J. R., Tout, C. A., \& Pols, O. R. 2002, MNRAS, 329, 897

Hurley, J. R. \& Shara, M. M. 2003, ApJ, 589, 179

Ivanova, N., Belczynski, K., Fregeau, J. M. \& Rasio, F. A. 2005, MNRAS, 358, 572

Ivanova, N., Heinke, C. O., Rasio, F. A., Taam, R. E., Belczynski, K. \& Fregeau, J. 2006, MNRAS, 372, 1043

Ivanova, N., Heinke, C. O., Rasio, F. A., Belczynski, K. \& Fregeau, J. 2007, MNRAS, submitted (astro-ph/0706.4096v1) 
Joshi, K. J., Nave, C. P., Rasio, F. A. 2001, ApJ, 550, 691

Kim D.-W., Fabbiano G., 2004, ApJ, 611, 846

Kim, C., Kalogera, V., Lorimer, D. R., Ihm, M. \& Belczynski, K. 2005, ASP, 328, 83

King, I. R. 1965, AJ, 70, 376

Kroupa, P. 2002, Science, 295, 82

Kulkarni, S., Hut, P., \& McMillan, S. 1993, Nature, 364, 421

Mackey, A. D., Wilkinson, M. I., Davies, M. B. \& Gilmore, G. F. 2007, MNRAS, 397, 40L

Meylan, G., Heggie, D. C. 1997, A\&ARv, 8, 1

Merritt, D., et al. 2004, ApJ, 608, L25

Miller, M. \& Hamilton D. 2002, MNRAS, 330, 232

O’Leary, R., O’Shaughnessy, R., \& Rasio, F.A. 2007, to appear in PRD Rapid Communications (arXiv:astro-ph/0701887)

O’Leary, R. M., Rasio, F. A., Fregeau, J. M., Ivanova, N., O’Shaughnessy, R. 2006, ApJ, 637, 937

O’Shaughnessy, R., Kim, C., Kalogera, V., \& Belczynski, K. 2006, ApJ, submitted (astro-ph/0610076)

Pfahl, E., Podsiadlowski, P. \& Rappaport, S. 2005, ApJ, 628, 343

Phinney, S. 1991, ApJ, 380, L17

Portegies Zwart, S. F., \& McMillan, S. 2000, ApJ, 528, L17

Portegies Zwart, S. F., \& Meinen, A. T. 1993, A\&A, 280, 174

Sigurdsson, S., \& Hernquist, L. 1993, Nature, 364, 423

Sopuerta, C. F., Yunes, N., Laguna P. 2007, ApJL, 656, 9

Vesperini, E. 1998, MNRAS, 299, 1019

Voss, R., Tauris, T. M. 2003, MNRAS, 342, 1169

Watters, W. A., Joshi, K. J., Rasio, F. A. 2000, ApJ, 539, 331

Webbink, R. F. 1984, ApJ, 277, 355

Webbink, R. F. 1985, in IAU Symp. 113: Dynamics of Star Clusters, 541-577

Woitas, J., Leinert, C., \& Kohler, R. 2001, A\&A, 376, 982 


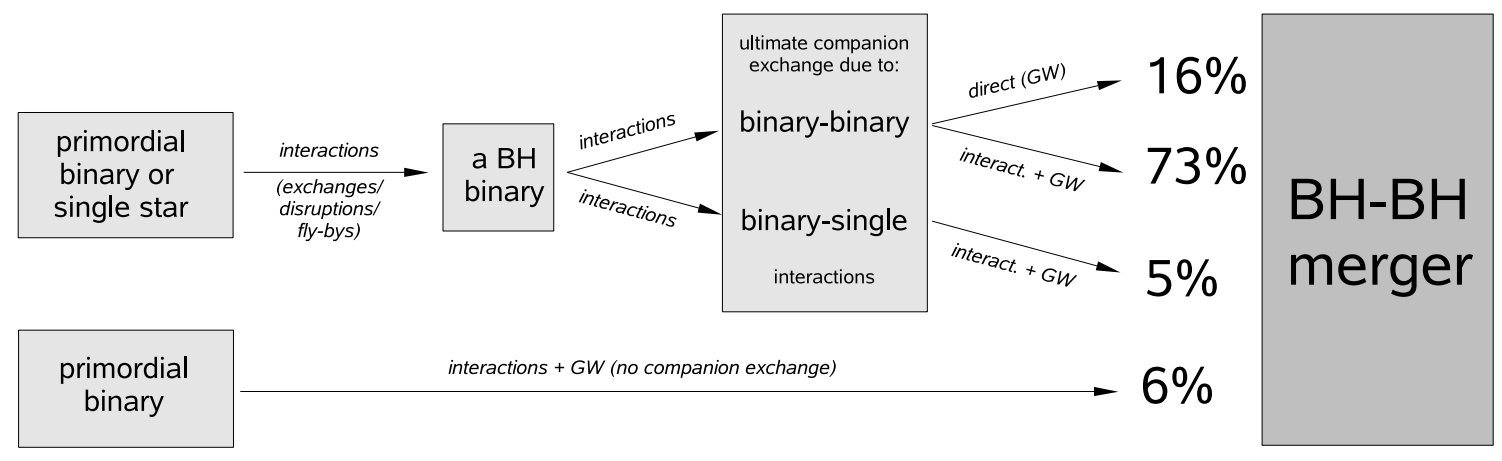

Fig. 1.- Channels leading to BH-BH mergers in clusters with their contributions. 'Interactions' means any kind of dynamical interactions with the exception of the primordial BH binary channel where no companion exchange takes place; 'GW' means binary tightening due to gravitational wave emission. For details see 33.1 .

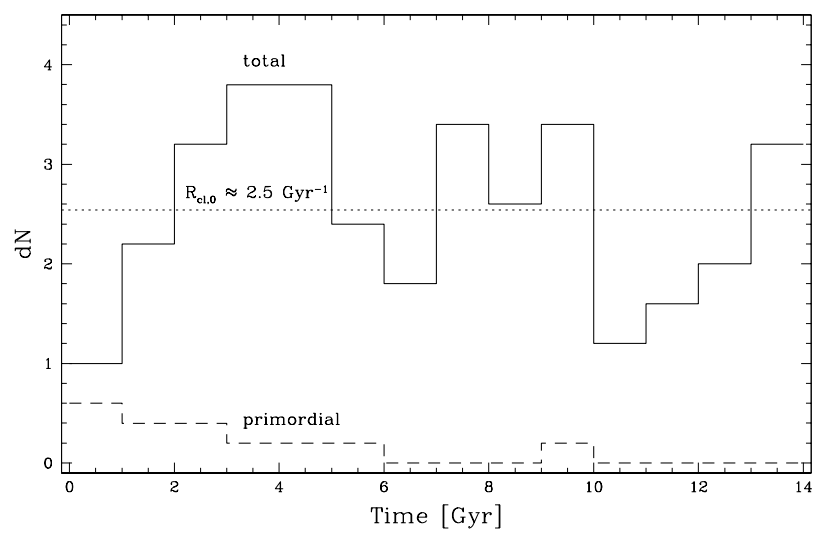

Fig. 2.- Number of $\mathrm{BH}-\mathrm{BH}$ mergers versus time averaged over 5 independent runs $\left(M_{\text {cluster }} \approx 4.82 \times 10^{5} M_{\odot}\right)$. The dashed line shows the number of $\mathrm{BH}$ mergers coming from primordial $\mathrm{BH}$ binaries (the bottom-most channel in Fig 1). Note that all numbers were binned per Gyr, so the rate of BH-BH mergers $\left(G_{y r}^{-1}\right)$ can be read directly off this plot and the average (approximately constant) merger rate is indicated by the dotted line. 


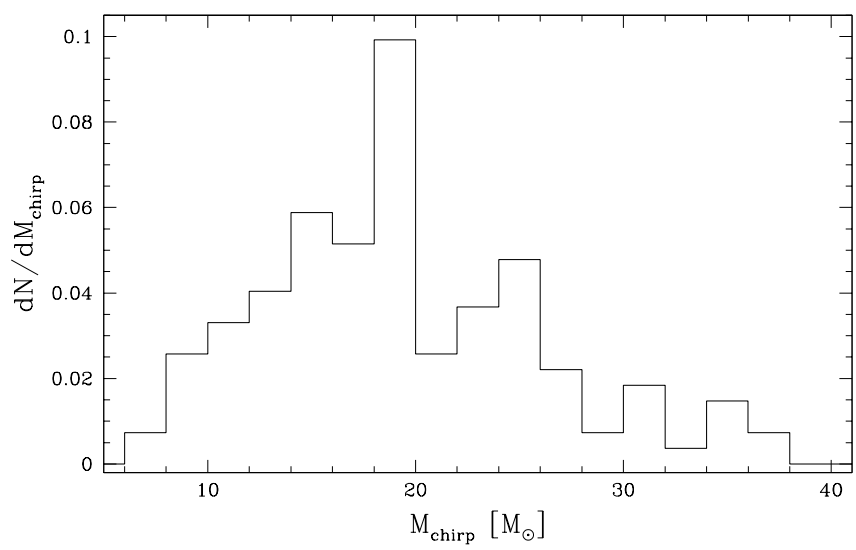

Fig. 3.- Distribution of chirp masses $\left(\left(M_{1} M_{2}\right)^{3 / 5}\left(M_{1}+M_{2}\right)^{-1 / 5}\right)$ of merging BH-BH binaries in the cluster population.

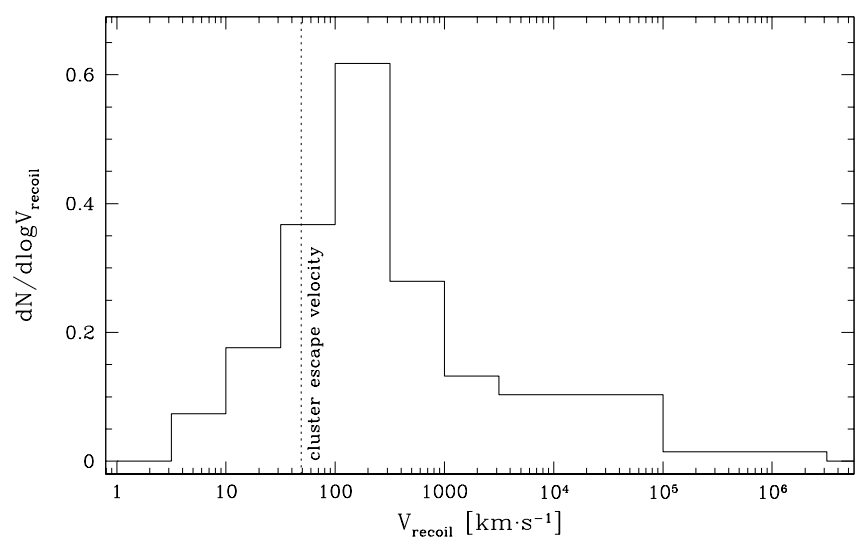

Fig. 4.- Distribution of recoil velocities obtained by $\mathrm{BH}-\mathrm{BH}$ mergers due to anisotropic GW emission. About $70 \%$ of BHs get kick velocities higher than the cluster escape speed. Note the horizontal axis logarithmic scale. 


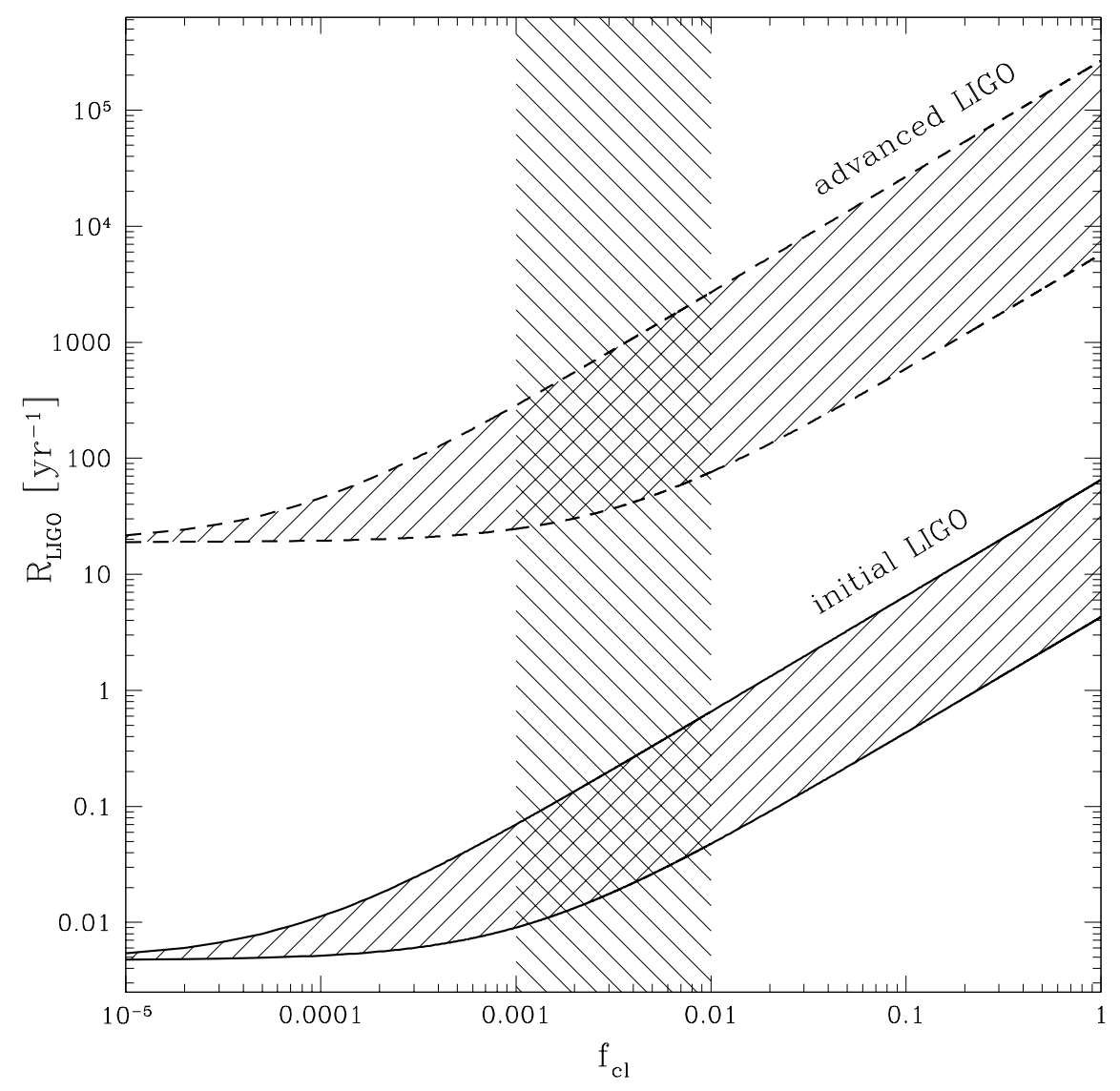

Fig. 5.- LIGO and Advanced LIGO detection rates of merging double compact binaries as a function of the assumed initial stellar mass fraction in dense star clusters. The vertical shaded area represents the most likely range of this parameter (see $\S 4$ for more details). For both versions of the LIGO detector two lines are shown: the lower limit is based on the cluster merger rates obtained by O'Leary et al. (2006) while the upper limit is based on the model described in this work. 